\title{
Enrolling Children in Clinical Trials for Genetic Neurodevelopmental Conditions: Ethics, Parental Decisions, and Children's Identities
}

\author{
Erin Turbitt, Ainsley J. Newson, Barbara B. Biesecker, and Benjamin S. Wilfond
}

ABSTRACT Knowledge of genetic mechanisms contributing to neurodevelopmental conditions is advancing. This is informing development of new drugs to treat or ameliorate these conditions, through targeting underlying genetic pathways. Drugs are tested in clinical trials, necessitating parents to engage with decisions about whether to enroll their child. In this article, we consider important ethical issues to anticipate as clinical research opportunities in genetic neurodevelopmental conditions arise. For example, genetic pathways targeted by the drugs may interact with valued character and personality traits. It is essential that recruitment and consent processes are optimized for families who will grapple with whether these novel drug treatments interact with their child's personality and authentic identity. We call for focused social science research and further normative analysis so that parents are better supported to make informed choices. Additionally, clinical research regulators should have a sound understanding of the contextual experiences regarding how this population of parents engages with decisions.

KEYWORDS human research ethics, decision-making for children and adolescents, research enrollment, informed consent, enhanced consent, clinical trials, pediatrics, neurodevelopmental disability, research equity, personhood Turbitt, E., A. J. Newson, B. B. Biesecker, and B. S. Wilfond, "Enrolling Children in Clinical Trials for Genetic Neurodevelopmental Conditions: Ethics, Parental Decisions, and Children's Identities," Ethics \& Human Research 43, no. 4 (2021): 27-36. D0l: 10.1002/eahr.500097

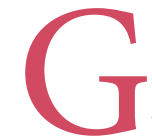
enomics is advancing the understanding of neurodevelopmental conditions such as intellectual disability, autism spectrum disorder, and global developmental delay. ${ }^{1}$ Despite these promising advances, genomics has not yet reached the treatment and management of neurodevelopmental conditions to the same extent as it has certain other specialties, such as oncology. ${ }^{2}$ One response that may address this disparity are new pharmaceutical treatments for genetic neurodevelopmental conditions. These treatments are now in development, with the aim to eliminate the underlying condition, rather than target discrete symptoms individually. Clinical research that advances to drug trials is a key step in the pathway from basic science to clinical care.
Clinical research into genetic neurodevelopmental conditions has increased the number of families being offered opportunities to enroll their child in clinical drug trials. It is therefore a good time to consider parents' decision-making processes, along with the recruitment and consent procedures with which parents must engage in order to enroll their child. Although we focus here mainly on parental decision-making for minors, much of what we discuss applies also to decision-making for adult children. The capacity to make decisions is often impaired in individuals with neurodevelopmental conditions, and their parents often must make decisions on their behalf for their entire life.

Research consent processes commonly rely on written study information. ${ }^{3}$ As clinical research increases in 
its complexity and reach, so too does the complexity of information to be communicated to prospective participants or, for minors and for many adults with limited capacity for independent decision-making, their parents. As a result, research enrollment materials are often extensive and exceed levels of general understanding. ${ }^{4}$ One problematic assumption of this approach (i.e., the focus on providing information) is that offering information fulfills the intent of the consent process. ${ }^{5}$

There are fewer opportunities for children, including those living with neurodevelopmental conditions, to participate in clinical research compared to opportunities for neurotypical adults. This is in part because past exploitation of children and those with neurodevelopmental conditions led to increased oversight and safeguards to protect these more vulnerable populations. Below, we discuss how a failure to appreciate the context in which decisions about research opportunities are made for individuals with genetic neurodevelopmental conditions, including minors and adults with reduced decisional capacity, may lead to marginalization and even exclusion of these populations from research. This is a timely consideration due to an increasing focus on equity and inclusivity in research (and in society more generally). We advocate for the importance of ensuring that those responsible for clinical research oversight and clinical investigators are aware of the considerations specific to people with these conditions.

Herein, we consider the importance of increasing clinical research opportunities for individuals with neurodevelopmental conditions, ensuring that those overseeing such research are appropriately aware of the context-specific considerations and potential barriers for these populations. We also discuss the negative implications of a narrow focus on information provision in the specific context of families of children who are not neurotypical (e.g., those living with genetic neurodevelopmental conditions) and suggest potential ways to mitigate these implications. We argue that recruitment and consent processes should be optimized for families of children with neurodevelopmental conditions.

We also introduce a further issue that parents may face: as new drug treatments aim to target the underlying mechanism of these conditions, parents may be concerned about the loss of positive aspects of their child's condition (such as their child's unique personality) along with the debilitating symptoms. This reflects a wider ethical issue: whether reversing neurodevelop-

\section{Table 1.}

\section{Challenges Raised by and Proposed Solutions for Enrolling Children in Clinical Trials for Genetic Neurodevelopmental Conditions}

\section{Challenges}

Investigators and IRBs may lack appreciation of the contextual experience of disabilities.

Informed consent processes tend to focus narrowly on information provision.

Parents and their children may have differing perspectives about trial enrollment and treatment.

Parents may have to reconcile themselves to the possibility that novel treatments may impact their child's personal identity and authenticity.

\section{Proposed solutions}

Investigators and IRBs should strive to be aware of and sensitive to families' circumstances.

Investigators and IRBs should reimagine the goals of informed consent to serve a range of functions.

Investigators could use interactive consent platforms.

Investigators could use narratives of adults with lived experiences of disability to increase parents' awareness.

Investigators could use digital support tools to enhance shared decision-making.

Further context-specific social science and normative research should be conducted, published, and critically discussed. 
mental conditions is justified in all cases. This broader issue is important, but here we will focus on the ethical issues that arise for parents as they consider whether to enroll their children in relevant trials.

We begin by describing neurodevelopmental conditions and developments in treatments for these conditions. We then critically review existing concerns over enrollment processes in research and suggest ways to address them (a summary is provided in the table). We focus on fragile $\mathrm{X}$ syndrome, which is the most common form of inherited intellectual disability. Among genetic conditions for neurodevelopmental conditions, the most treatment advances have been made for fragile $\mathrm{X}$ syndrome. We argue that enrolling children with genetic neurodevelopmental conditions into clinical trials raises novel ethical challenges due to the curative nature of these treatments, which target underlying pathways. We recommend further exploration in this area to inform the development of approaches to enrolling children into these drug trials that consider more dimensions of the contexts in which the families make such decisions.

\section{GENETIC NEURODEVELOPIMENTAL CONDITIONS}

$\mathrm{N}$ eurodevelopmental conditions are caused by brain impairments that present in childhood. ${ }^{6}$ The conditions overlap clinically, with many manifesting as cognitive deficits and behavioral challenges due to intellectual disability and autism. People with neurodevelopmental conditions are a heterogeneous group that includes individuals who lack capacity for independent decision-making throughout their lifespan. Therefore, as mentioned, parents often play a key role in making decisions on behalf of not only their minor but also their adult children with such conditions. ${ }^{7}$

Some neurodevelopmental conditions are identified abruptly due to an infection, trauma, or congenital malformation, while others develop more subtly, with developmental delays becoming apparent as a child grows older. In these latter cases, the precise cause is often unknown, although most are likely associated with pathogenic (i.e., disease-causing) genetic variants that may have been inherited or have spontaneously arisen in the individual.

Within genetics and medicine, there is a continuum of understanding about the genetic contribution to neurodevelopmental conditions across conditions. ${ }^{8}$ At one extreme, genetic causes are largely unknown, as in the case with attention deficit hyperactivity disorder (ADHD). While ADHD does appear to run in families, only a small subset of the associated genes have been identified to date.

At the center of the continuum are conditions such as autism spectrum disorder. Some genes associated with this condition have been identified, but many cases have no known genetic contribution, suggesting that the condition often results from an interplay of many

\section{As new drug treatments aim to target} the underlying mechanism of genetic neurodevelopmental conditions, parents may be concerned about the loss of positive aspects of their child's condition, such as their child's unique personality.

genes. ${ }^{9}$ Two predominant models have emerged to explain the genetics of autism spectrum disorder. Some cases are caused by one or a handful of strong-to-moderate disease-causing variants, whereas other cases are likely caused by many weak disease-causing variants, in combination with environmental factors. ${ }^{10}$

At the other end of the causal spectrum are conditions for which variation in a specific gene has been identified as the cause. An example is fragile $\mathrm{X}$ syndrome, the most commonly inherited cause of intellectual disability, associated with behavioral and learning challenges. As mentioned, we will focus on fragile $\mathrm{X}$ syndrome because of its frequency and the availability of clinical trials. ${ }^{11}$ However, the issues we explore are likely to be relevant to other genetic neurodevelopmental conditions for which pharmaceutical treatment may be available in the future. This includes conditions such as Angelman syndrome, 16p11.2 deletion or duplication syndrome, and many others that are increasingly 
identified and diagnosed as a result of improvements in genomic testing capabilities. ${ }^{12}$

As with many genetic neurodevelopmental conditions, there is a wide spectrum of clinical features among individuals with fragile $\mathrm{X}$ syndrome. Behavioral symptoms include anxiety, aggression, attention deficits, hyperactivity, and autism. ${ }^{13}$ Cognitive and social impairments occur in most males with fragile X syndrome, while these deficits are significantly milder in females with this condition. Individuals with fragile $\mathrm{X}$ syndrome often have distinct personality traits such as a cheerful disposition and sense of humor. ${ }^{14}$ Mothers of children with fragile $\mathrm{X}$ syndrome who are carriers of the condition have a higher incidence of social anxiety and depressive symptoms than is found in the general population. ${ }^{15}$

Existing pharmaceutical treatments for fragile $\mathrm{X}$ syndrome target discrete symptoms such as inattention, hyperactivity, or aggressive behaviors. Genomics has advanced understanding of the underlying cause of several neurodevelopmental conditions, in particular fragile X syndrome, leading to a new generation of potential treatments and interventions that target the underlying molecular pathway of the condition. ${ }^{16} \mathrm{New}$, experimental treatments for fragile $\mathrm{X}$ syndrome (such as mGluR5 negative allosteric modulators, or NAMs) aim to address underlying brain dysfunction and provide global symptom reduction, including improvement of cognitive and learning impairment. ${ }^{17}$ The goal of these treatments is to provide what is essentially an irreversible cure for the condition. While treatments like NAMs are not yet available, clinical trials aiming to demonstrate their effectiveness (mostly phase II and some phase III) are underway. ${ }^{18}$ Additionally, it is hoped that the advances in fragile $\mathrm{X}$ syndrome drug development will inform advances in treatment for other genetic neurodevelopmental conditions. ${ }^{19}$

\section{PEDIATRIC CLINICAL RESEARCH AND ENROLLIMENT DECISIONS}

$\mathrm{P}$ ediatric clinical research is essential to advances in treatment of neurodevelopmental conditions, as generalizing evidence from adults to pediatric patients is an inadequate approach. For example, children's developing brains may react differently from those of fully developed adults. ${ }^{20}$ In the mid-twentieth century, efforts to enhance research regulation (and through this to protect children against exploitation in research) culminated in the development of guidelines such as the World Medical Association's Declaration of Helsinki and The Belmont Report in the United States. ${ }^{21}$

International guidelines were key to establishing an essential discourse on pediatric clinical research. Yet a delicate balance exists between protecting children in research and ensuring that protections do not unnecessarily obstruct development of pediatric-specific drug safety and efficacy data. Efforts to increase pediatricspecific data serve both scientific (improved knowledge) and ethical (distributive justice) purposes. ${ }^{22}$ But a wider problem remains regarding the underrepresentation of many groups (including children, people with disabilities, women, and people of color) in pharmaceutical research.

While there has been an increase in pediatric clinical research in recent decades, a 2012 report showed that, overall, a discrepancy remains; there were 10 times more clinical trials for adults than for children. ${ }^{23}$ Of the pediatric trials that are initiated, $19 \%$ are discontinued, often due to low recruitment and poor retention of participants. ${ }^{24} \mathrm{~A}$ more recent analysis of clinical trials concerning rare diseases reported that $12 \%$ of the trials focused on the pediatric population; within that study, a discontinuation rate of $30 \%$ was found in the pediatric trials. ${ }^{25}$ The comparatively smaller number of pediatric clinical trials among clinical trials in general is, in part, attributable to historical events in pediatric research, where children were exploited or harmed. ${ }^{26}$

Mitigation attempts in response to this continuing underrepresentation include recent legislative advances such as the Best Pharmaceuticals for Children Act in the United States, which is accelerating the accumulation of pediatric-specific drugs. ${ }^{27}$ As new treatments for neurodevelopmental conditions are developed, there is an expanding need for children to be enrolled in clinical drug trials to amass evidence on high-quality safety and dosage information.

There are further considerations with respect to enrolling children with neurodevelopmental conditions into clinical drug trials. Families of children with these conditions have historically been underserved by and disenfranchised and often excluded from research opportunities. ${ }^{28}$ Recent evidence shows that parents want 
their children to participate, although they may face barriers beyond those faced by families of neurotypical children. ${ }^{29}$ These barriers include challenges in determining whether a child with a neurodevelopmental condition can contribute to decisions about enrollment and challenges for the parent in engaging with such decisions while experiencing many other demands associated with caring for a child with a neurodevelopmental condition. Among families of children with neurodevelopmental conditions, those that report greater stress are less likely to join a clinical research registry. ${ }^{30}$ This is of particular relevance to families of children with fragile $\mathrm{X}$ syndrome, in which (as mentioned) female carrier status is associated with higher risks for social anxiety and depressive symptoms. ${ }^{31}$

For any research involving children, regulations generally require parental permission and, if the child is capable, their affirmative agreement (discussed further below). ${ }^{32}$ These decisions can be difficult for parents, particularly as risks and benefits can be highly subjective. Institutional review boards (IRBs) and the research team have prominent roles in determining whether specific research involving children is acceptable. Freedman and coauthors introduce the related concept in loco parentis to describe scenarios whereby studies that may pose an unacceptable risk to children are evaluated by the research team and IRBs and filtered out early, prior to reaching the stage that requires parental permission. ${ }^{33}$

For a concept such as in loco parentis to function, investigators need to have a thorough working knowledge of the target population, and IRBs tasked with approving such research should be flexible and able to understand families' perspectives on their child's participation in research. Sabatello and coauthors warn that IRBs may inadvertently be overly restrictive and unnecessarily exclude individuals with disabilities (including those with neurodevelopmental conditions) from participating in clinical research. In an attempt to mitigate such risks, they describe the importance of what they term "disability cultural competence" for those working with patients and families with disability who are enrolled or considering enrolling in clinical research. ${ }^{34}$ Such training, awareness, and sensitivity can positively contribute to enabling inclusivity in research. Investigators developing drugs for genetic neurodevelopmental conditions and IRB members approving protocols to test these drugs thus have a responsibility to ensure that they are knowledgeable about the circumstances of these families and how to best support them to make decisions about clinical trial enrollment. Cascio and colleagues recently conducted a literature review to highlight strategies that can be employed to ensure that research involving individuals with autism spectrum disorder can respect the needs of both the individual participant and the group to advance knowledge about the condition. They argue for a "person-orientated research ethics framework" that is flexible to meet the needs of individuals with the disorder. ${ }^{35}$ Such a framework could be used when designing and conducting research involving children with genetic neurodevelopmental conditions.

\section{CURRENT ISSUES WITH CONSENT PROCESSES}

The consent processes in medical research are increasingly recognized as problematic. ${ }^{36}$ The concept of informed consent emphasizes participants' understanding of key relevant information. However, it is challenging to determine how much information a potential participant needs to make an informed decision. Many participants fail to understand basic concepts about the research in which they are enrolled. ${ }^{37}$ As medical research has become increasingly sophisticated, the length and complexity of information provided to participants during the consent process has increased. ${ }^{38}$ Focusing too narrowly on information also potentially neglects other relevant aspects of research participation, such as ensuring that decisions reflect the values of the decision-maker. A possible solution to these challenges lies in adopting a more holistic approach to the goals of informed consent, one that reaches beyond simply informing participants.

The challenge of information provision and the value of moving away from a conceptualization of consent that focuses too narrowly on providing information are particularly relevant to genetic neurodevelopmental conditions. Beyond the features common to clinical trial research for the wide range of health conditions (e.g., randomization, placebos, and the risk of side effects), trials for fragile X syndrome treatments may have the added complexity of targeting genetic pathways. ${ }^{39}$ It is widely documented that levels of genetic literacy are low across the public at large. ${ }^{40}$ This added layer of com- 
plexity that may need to be conveyed to families could further impede an informed consent process that relies too heavily on information provision. There are efforts underway in other disease contexts to reduce the complexity of the informed consent process. ${ }^{41}$ However, assessing the effectiveness of these efforts has thus far been limited to studies of relatively well-educated adults making decisions about their own participation in research. ${ }^{42}$

One approach to the problem of the increasing complexity of information about trials is to critically consider the roles that information provision and understanding should play in consent processes. Beskow and colleagues, for example, suggest that understanding is an "ethical aspiration" rather than a requirement for enrollment in research. ${ }^{43}$ In their recent empirical study, their participants (who were experts such as bioethicists, geneticists, social scientists, and community/ patient representatives) were confronted with the reality that many adults would be excluded from research if required to reach a particular threshold of understanding about study information. ${ }^{44}$ Therefore, the expert participants adjusted their opinions about what should be considered required understanding.

Further, when information is provided during study enrollment, this communication generally consists of a unidirectional process of information provision. Adult learning theory and education research indicate that merely having information relayed to oneself is one of the most ineffective ways of learning. ${ }^{45}$ One response to this could be to reduce the emphasis on providing detailed information within the consent process and to increase efforts that focus on parents' concerns and feelings about the research. This is particularly pertinent to consider for enrollment in fragile $\mathrm{X}$ syndrome trials, given the added layer of complexity associated with the experimental treatments.

Informed consent should also be conceptualized beyond the one-off interaction that occurs when an investigator provides study information to the potential participant or their proxy decision-maker and this person agrees to or declines enrollment. Informed consent should instead be seen to serve a variety of functions, which occur across a range of interactions and time points. Dickert and colleagues describe many functions of consent, including alignment of participant values with their enrollment decisions, promotion of trust, and protection of the welfare of participants. ${ }^{46}$ These specific functions may be particularly relevant to trials involving children with neurodevelopmental conditions, and they relate closely to points raised earlier about why it is important for investigators and IRB members to appreciate the contexts of these families and to develop disability cultural competencies.

There are also practical tools that can be employed to reframe informed consent as a dynamic process beyond information provision in a one-off interaction. For example, interactive consent platforms and decision aids that promote values clarification can be developed and tested. ${ }^{47}$ These tools may have particular value in the context of enrolling children with neurodevelopmental conditions. Consider the reality of traditional consent interactions with families in which a child has fragile $\mathrm{X}$ syndrome. If the child is present, they may be disruptive and anxious, especially in an unfamiliar environment, impacting parents' ability to concentrate and absorb necessary information. This is coupled with the potential for the mother to have symptoms of anxiety as part of her carrier phenotype, which can make these types of decisions particularly challenging.

A particular consideration for informed consent in the context of genetic neurodevelopmental clinical trials is whether parents, especially if not themselves diagnosed with the condition in question, appreciate what is in their specific child's best interest. While there is no doubt that parents are likely to do what they think is in the best interest of their child, it may not be possible to determine what is in the child's best interest without having the experience of being affected by the condition. Further, for diagnosed individuals who choose to actively participate in decisions about trial enrollment, and have the capacity to do so, there is the potential for choice discrepancies to arise between parents and children. In the context of treatment options for autism spectrum disorder, for example, Perry suggests that parents may lack the intimate insight their child possesses through their lived experience with the condition. ${ }^{48}$ A solution Perry provides for the possible disconnect between parents' and children's perspectives is to offer parents access to narratives from adults with autism spectrum disorder. Such narratives may be effective in contributing to parents' understanding of the lived ex- 
perience of autism spectrum disorder and inform their decision-making about treatments, including enrollment in clinical drug trials. We endorse this suggestion and encourage further investigation into its wider use across other neurodevelopmental conditions.

Additionally, soliciting the potential research participant's preference can be useful and important. As we have discussed, there is a wide spectrum of symptom severity among individuals with genetic neurodevelopmental conditions. Even with a relatively well-described condition such as fragile $\mathrm{X}$ syndrome, individuals vary widely in cognitive functioning from mildly to severely affected. Digital support tools may provide an interactive and accessible option for adolescents and adults with limited decisional capacity to participate in the consent processes, enhancing shared decision-making between affected individuals and their parents. ${ }^{49}$ These tools use interactive multimedia (audio, visual, and narration) with features such as an avatar that walks participants through the consent process, sorting exercises to engage participants in the decision, and knowledge checks.

In summary, while families of children with neurodevelopmental conditions may be at increased risk of experiencing the limitations of current informed consent processes, this could be somewhat mitigated by reducing consent processes' focus on information provision and through the use of tools to support both parents and potential participants who are engaged in enrollment decisions. This will, in turn, promote other important components of trial participation, including alignment with participant values and trust in both the researchers and the IRB process.

\section{NOVEL CHALLENGES FOR PARENTS}

necdotally, there is widespread support from the A fragile $\mathrm{X}$ syndrome advocacy community for developing the novel treatments we have described above. ${ }^{50}$ To advance these efforts, engagement with patient and community stakeholders in identifying treatment priorities is key to the drug development process and indeed is endorsed by the U.S. Food and Drug Administration's Patient-Focused Drug Development initiative. $^{51}$

Despite this widespread support, parents of children with fragile $\mathrm{X}$ syndrome nevertheless have to reconcile their thoughts about which attributes of the syndrome they wish to see reduced in their child with their ideas about which others they may perceive to be authentic aspects of their child's character or personal identity, of "who their child is", and thus important to preserve. From research in a comparable context, we know that parents of children with Down syndrome have expressed concerns that a cure for Down syndrome could change their child's personality. ${ }^{52}$ Like individuals with Down syndrome, those with fragile $\mathrm{X}$ syndrome often have cheerful, pleasant personalities. ${ }^{53}$

Further parallels could be drawn from discourse about the use of stimulants such as Ritalin (methylphenidate) or Adderall (amphetamine/dextroamphetamine) in children living with ADHD. Hinshaw et al. raise concerns that the use of these drugs may threaten one's authenticity or mask one's "true self." ${ }^{4}$ Empirical work by Singh, however, suggests that, from the child's perspective, while psychotropic drugs do impact their behavior, concerns about threats to their authenticity were unfounded. ${ }^{55}$

Nevertheless, there are two points of difference between pharmaceutical treatment for fragile $\mathrm{X}$ syndrome compared to for ADHD. First, drug interventions for ADHD target the behavioral symptoms of the condition, whereas the drugs in development for fragile $\mathrm{X}$ syndrome target the underlying cause (with a view to improving both behavioral and cognitive symptoms). Second, these fragile $\mathrm{X}$ syndrome drugs aim to provide what is essentially an irreversible cure for the condition, whereas drugs such as Ritalin temporarily target behavioral symptoms with no or few long-term implications and the possibility to stop treatment at any time. There may be value in replicating empirical work, such as that done by Singh, with families of children with fragile $\mathrm{X}$ syndrome. A recent qualitative study by Boardman explored views about population screening programs for fragile $\mathrm{X}$ syndrome by interviewing affected families, including two individuals diagnosed with the syndrome. ${ }^{56}$ While not the primary focus of the study, one theme incorporated concepts about identity. Boardman documented disparate views from parents of affected individuals about whether a distinction between a person and their condition was possible in this context.

Empirical data are scarce about parents' perspectives on the impact on the child's personality of novel 
medications to treat fragile $\mathrm{X}$ syndrome. A qualitative study by D'Amanda and colleagues with 34 parents of children with fragile $\mathrm{X}$ syndrome found that personality strengths such as happiness, kindness, and compassion were perceived as the most positive aspects of their child's condition. ${ }^{57}$ These investigators also found that the majority of parents were not concerned that these personality strengths could be affected by an experimental drug treating the underlying condition. However, a small number of parents voiced some concerns about such drugs "removing the blindfold," in that fragile X syndrome allows their child to live a sheltered and relatively carefree life. ${ }^{58}$ These data indicate that further context-specific evidence may be warranted and that ongoing engagement with stakeholder communities, to better understand the priorities of parents and families, will continue to be key to successful treatment trials. In parallel, further normative exploration of concepts such as authenticity in the context of fragile $\mathrm{X}$ syndrome and other genetic neurodevelopmental conditions is important to increase understanding of the implications of the availability of a pharmaceutical cure.

\section{CONCLUSION}

W ith the increasing number of clinical trials that target genetic conditions with behavioral and cognitive impairments, more parents with children living with these conditions will face difficult decisions about their willingness to enroll their children in these trials. There are limitations to current consent and recruitment processes that impact these families. To help overcome these challenges and work toward solutions that promote inclusivity, engaging with people with genetic neurodevelopmental conditions and their parents will be key (see the table). Furthermore, ensuring "disability cultural competence" among IRBs and regulators overseeing clinical research may help to ensure these populations benefit from such research. ${ }^{59}$ Even if we reach a stage where these processes are optimized for all stakeholders, parents will still (with appropriate support) need to reflect on their own values and perceptions about whether treatments targeting the underlying mechanism of genetic neurodevelopmental conditions could impact their child's personhood and on their level of comfort around that notion.
Erin Turbitt, PhD, is a lecturer in the Graduate School of Health at the University of Technology Sydney; Ainsley J. Newson, PhD, is a professor of bioethics at Sydney Health Ethics in the Faculty of Medicine and Health at the University of Sydney; Barbara B. Biesecker, PhD, is a Distinguished Fellow at RTI International in Washington DC; and Benjamin S. Wilfond, MD, is a professor of pediatrics at the University of Washington and Seattle Children's Research Institute.

\section{ACKNOWLEDGMENT}

Thank you to the parents who participated in our research about clinical trial decision-making and brought the issues discussed here to our attention.

\section{REFERENCES}

1. Tărlungeanu, D. C., and G. Novarino, "Genomics in Neurodevelopmental Disorders: An Avenue to Personalized Medicine," Experimental and Molecular Medicine 50, no. 8 (2018): doi:10.1038/s12276-018-0129-7.

2. Moreno-De-Luca, D., M. Ross, and D. Ross, "Leveraging the Power of Genetics to Bring Precision Medicine to Psychiatry: Too Little of a Good Thing?," Biological Psychiatry 83 (2018): e45-e46.

3. Jefford, M., and R. Moore, "Improvement of Informed Consent and the Quality of Consent Documents," Lancet Oncology 9 (2008): 485-93.

4. Grady, C., "Enduring and Emerging Challenges of Informed Consent," New England Journal of Medicine 372 (2015): 855-62.

5. O’Neill, O., “Some Limits of Informed Consent," Journal of Medical Ethics 29, no. 1 (2003): 4-7.

6. American Psychiatric Association, Diagnostic and Statistical Manual of Mental Disorders: DSM-5 (Arlington, VA: American Psychiatric Association, 2013).

7. D’Amanda, C., et al., "Fragile X Syndrome Clinical Trials: Exploring Parental Decision Making," Journal of Intellectual Disability Research 63 (2019): 926-35.

8. Blesson, A., and J. S. Cohen, "Genetic Counseling in Neurodevelopmental Disorders," Cold Spring Harbor Perspectives in Medicine 10, no. 4 (2020): doi:10.1101/cshperspect.a036533.

9. Vorstman, J. A., et al., "Autism Genetics: Opportunities and Challenges for Clinical Translation," Nature Reviews Genetics 18 (2017): 362-76.

10. de la Torre-Ubieta, L., et al., "Advancing the Understanding of Autism Disease Mechanisms through Genetics," Nature Medicine 22 (2016): 345-61.

11. Hagerman, R. J., and P. J. Hagerman, Fragile X Syndrome: Diagnosis, Treatment, and Research, 3rd ed. (Baltimore, MD: Johns Hopkins University Press, 2002).

12. Blesson and Cohen, "Genetic Counseling in Neurodevel- 
opmental Disorders."

13. Hagerman and Hagerman, Fragile X Syndrome.

14. Maes, B., et al., "Phenotypic Checklist to Screen for Fragile X Syndrome in People with Mental Retardation," Mental Retardation 38, no. 3 (2000): 207-15.

15. Hagerman, R. J., et al., "Fragile X-Associated Neuropsychiatric Disorders (FXAND)," Frontiers in Psychiatry 9 (2018): doi:10.3389/fpsyt.2018.00564; Movaghar, A., et al., "Data-Driven Phenotype Discovery of FMR1 Premutation Carriers in a Population-Based Sample," Science Advances 5, no. 8 (2019): doi:10.1126/sciadv.aaw7195.

16. Berry-Kravis, E. M., et al., "Drug Development for Neurodevelopmental Disorders: Lessons Learned from Fragile X Syndrome," Nature Reviews Drug Discovery 17 (2018): 28098.

17. Gross, C., et al., "Therapeutic Strategies in Fragile X Syndrome: From Bench to Bedside and Back," Neurotherapeutics 12 (2015): 584-608.

18. Tărlungeanu and Novarino, "Genomics in Neurodevelopmental Disorders."

19. Berry-Kravis, "Drug Development for Neurodevelopmental Disorders."

20. Kimland, E., and V. Odlind, "Off-Label Drug Use in Pediatric Patients," Clinical Pharmacology \& Therapeutics 91 (2012): 796-801.

21. Declaration of Helsinki, World Medical Association, October 2000, https://www.wma.net/policies-post/wma-declaration-of-helsinki-ethical-principles-for-medical-researchinvolving-human-subjects/; National Commission for the Protection of Human Subjects of Biomedical and Behavioral Research, The Belmont Report: Ethical Principles and Guidelines for the Protection of Human Subjects of Research (Washington, DC: U.S. Government Printing Office, 1979).

22. List, J. M., "Histories of Mistrust and Protectionism: Disadvantaged Minority Groups and Human-Subject Research Policies," American Journal of Bioethics 5, no. 1 (2005): 53-56.

23. Bourgeois, F. T., et al., "Pediatric versus Adult Drug Trials for Conditions with High Pediatric Disease Burden," Pediatrics 130 (2012): 285-92.

24. Pica, N., and F. Bourgeois, "Discontinuation and Nonpublication of Randomized Clinical Trials Conducted in Children," Pediatrics 138 (2016): e20160223.

25. Rees, C. A., et al., "Noncompletion and Nonpublication of Trials Studying Rare Diseases: A Cross-Sectional Analysis," PLoS Medicine 16, no. 11 (2019): e1002966.

26. Diekema, D. S., "Conducting Ethical Research in Pediatrics: A Brief Historical Overview and Review of Pediatric Regulations," Journal of Pediatrics 149, supplement 1 (2006): S3-S11.

27. Avant, D., G. T. Wharton, and D. Murphy, "Characteristics and Changes of Pediatric Therapeutic Trials under the Best
Pharmaceuticals for Children Act," Journal of Pediatrics 192 (2018): 8-12.

28. Feldman, M., et al., "Where Are Persons with Intellectual Disabilities in Medical Research? A Survey of Published Clinical Trials," Journal of Intellectual Disability Research 58 (2014): 800-809.

29. Kalb, L., et al., "Interest in Research Participation among Caregivers of Children with Neurodevelopmental Disorders," Journal of Autism and Developmental Disorders 49 (2019): 3786-97.

30. Ibid.

31. Hagerman, "Fragile X-Associated Neuropsychiatric Disorders (FXAND)"; Movaghar, "Data-Driven Phenotype Discovery."

32. American Academy of Pediatrics Committee on Bioethics, "Informed Consent, Parental Permission, and Assent in Pediatric Practice," Pediatrics 95 (1995): 314-17.

33. Freedman, B., et al., "In Loco Parentis. Minimal Risk as an Ethical Threshold for Research upon Children," Hastings Center Report 23, no. 2 (1993): 13-19, at 18.

34. Sabatello, M., "Cultivating Inclusivity in Precision Medicine Research: Disability, Diversity, and Cultural Competence," Journal of Community Genetics 10 (2019): 363-73, at 364.

35. Cascio, M. A., J. A. Weiss, and E. Racine, "Person-Oriented Research Ethics to Address the Needs of Participants on the Autism Spectrum," Ethics \& Human Research 42 (2020): 2-16, at 10 .

36. Grady, C., et al., "Informed Consent," New England Journal of Medicine 376 (2017): 856-67; Henderson, G. E., "Is Informed Consent Broken?", American Journal of the Medical Sciences 342 (2011): 267-72.

37. Falagas, M. E., et al., "Informed Consent: How Much and What Do Patients Understand?," American Journal of Surgery 198 (2009): 420-35.

38. Albala, I., M. Doyle, and P. S. Applebaum, "The Evolution of Consent Forms for Research: A Quarter Century of Changes," IRB: Ethics \& Human Research 32, no. 3 (2010): $7-11$.

39. Lea, D. H., et al., "Communicating Genetic and Genomic Information: Health Literacy and Numeracy Considerations," Public Health Genomics 14 (2011): 279-89.

40. Chapman, R., et al., "New Literacy Challenge for the Twenty-First Century: Genetic Knowledge Is Poor Even among Well Educated," Journal of Community Genetics 10 (2019): 73-84.

41. Spellecy, R., et al., "Easy-to-Read Informed Consent Form for Hematopoietic Cell Transplantation Clinical Trials: Results from the Blood and Marrow Transplant Clinical Trials Network 1205 Study," Biology of Blood and Marrow Transplantation 24 (2018): 2145-51. 
42. Turbitt, E., et al., "A Randomized Controlled Study of a Consent Intervention for Participating in an NIH Genome Sequencing Study," European Journal of Human Genetics 26 (2018): 622-30; Grady, C., et al., "A Randomized Trial Comparing Concise and Standard Consent Forms in the Start Trial," PLoS One 12 (2017): e0172607.

43. Beskow, L. M., and K. P. Weinfurt, "Exploring Understanding of 'Understanding': The Paradigm Case of Biobank Consent Comprehension," American Journal of Bioethics 19, no. 5 (2019): 6-18, at 16.

44. Ibid.

45. Knowles, M. S., The Modern Practice of Adult Education: From Pedagogy to Andragogy (Englewood Cliffs, NJ: Cambridge Adult Education, 1980).

46. Dickert, N. W., et al., "Reframing Consent for Clinical Research: A Function-Based Approach," American Journal of Bioethics 17, no. 12 (2017): 3-11.

47. Kaye, J., et al., "Dynamic Consent: A Patient Interface for Twenty-First Century Research Networks," European Journal of Human Genetics 23, no. 2 (2015): 141-46.

48. Perry, A., "Autism beyond Pediatrics: Why Bioethicists Ought to Rethink Consent in Light of Chronicity and Genetic Identity”, Bioethics 26 (2012): 236-41.

49. McCormack, L. A., et al., "Supporting Informed Clinical Trial Decisions: Results from a Randomized Controlled Trial Evaluating a Digital Decision Support Tool for Those with Intellectual Disability”, PLoS One 14 (2019): e0223801.
50. A sense of this support is based on the authors' engagement with this community.

51. "Learn about FDA Patient Engagement," U.S. Food and Drug Administration, December 20, 2020, https://www.fda. gov/patients/learn-about-fda-patient-engagement.

52. Inglis, A., et al., "A 'Cure' for Down Syndrome: What Do Parents Want?”, Clinical Genetics 86 (2014): 310-17.

53. Maes et al., "Phenotypic Checklist to Screen for Fragile X Syndrome."

54. Hinshaw, S. P., and R. M. Scheffler, The ADHD Explosion: Myths, Medication, Money, and Today's Push for Performance (Oxford: Oxford University Press, 2014).

55. Singh, I., "Not Robots: Children's Perspectives on Authenticity, Moral Agency and Stimulant Drug Treatments," Journal of Medical Ethics 39 (2013): 359-66.

56. Boardman, F. K., "Attitudes toward Population Screening among People Living with Fragile X Syndrome in the UK: 'I Wouldn't Wish Him Away, I'd Just Wish His Fragile X Syndrome Away," Journal of Genetic Counseling 30, no. 1 (2021): 85-97.

57. D’Amanda, C., "Parental Decision Making for Fragile X Syndrome Clinical Trials" (master's thesis, Johns Hopkins University, 2017), at https://jscholarship.library.jhu.edu/handle/1774.2/40731.

58. D’Amanda, Parental Decision Making.

59. Sabatello, "Cultivating Inclusivity in Precision Medicine Research," 364. 